The BMJ

Cite this as: BMJ 2022;376:0117 http://dx.doi.org/10.1136/bmj.o117 Published: 17 January 2022

\section{Covid-19: Severe complications during pregnancy are more common in unvaccinated women, study finds}

\author{
Elisabeth Mahase
}

Unvaccinated women accounted for $77 \%$ of SARS-CoV-2 infections that have occurred during pregnancy in Scotland and 98\% of infections that led to a critical care admission, a study has found. ${ }^{1}$

Researchers looked at 4950 confirmed infections in pregnant women from 1 December 2020-when routine SARS-CoV-2 testing of maternity admissions began-which were spread relatively evenly throughout pregnancy (1543 in the first trimester, 1850 in the second, and 1557 in the third).

They found that "severe complications," such as critical care admission, stillbirths, and early neonatal deaths, were more common in those who were unvaccinated compared with those who were vaccinated.

Commenting on the study, Asma Khalil, professor of obstetrics and maternal fetal medicine at St George's University of London, said, "The study shows that the overwhelming majority of pregnant women and babies becoming unwell or dying as a result of covid-19 were unvaccinated. The pandemic is far from over and with tens of thousands of covid-19 cases still being reported in the UK every day, it is paramount that pregnant women continue to take up the offer of a vaccine."

Compared with non-pregnant women of reproductive age, pregnant women with SARS-CoV-2 infection are more likely to be admitted to critical care, receive invasive ventilation and extracorporeal membrane oxygenation, and die. ${ }^{2}$ Covid-19 during pregnancy has also been associated with an increased risk of pregnancy specific complications such as pre-eclampsia, preterm birth, and stillbirth. ${ }^{3}$

\section{Low uptake}

Despite this, the paper, published in Nature Medicine, reported that vaccination coverage (between 8 December 2020 and 31 October 2021) is substantially lower in pregnant women than in the general female population of 18 to 44 year olds, with just under a third (32.3\%) of women giving birth in October 2021 in Scotland having two doses of vaccine, compared with $77.4 \%$ in the general population group.

Of the 4950 confirmed infections during pregnancy, $823(17 \%)$ were associated with any hospital admission and 104 (2\%) with a critical care admission.

In terms of vaccination status, over three quarters of infections during pregnancy $(77 \%)$ occurred in women who were unvaccinated at the date of infection onset, with 12\% (567) in partially vaccinated women and $11 \%$ (550) in fully vaccinated women.

Of the infections in unvaccinated women, nearly one fifth (748 of 3833) were associated with hospital admission, compared with 8\% (47 of 567) for those who were partially vaccinated, and $5 \%$ ( 28 of 550 ) for fully vaccinated women.

\section{Perinatal deaths}

Of the total of 2364 babies that were born to women who had SARS-CoV-2 infection during pregnancy, there were 241 preterm births, 11 stillbirths, and eight neonatal deaths. This equates to a preterm birth rate of $10 \%$ and an extended perinatal mortality of 8 per 1000 births following SARS-CoV-2 infection at any point in pregnancy.

Looking at outcomes within 28 days of SARS-CoV-2 infection (620 births), there were 101 preterm births, 10 stillbirths, and four neonatal deaths, giving a preterm birth rate of $17 \%$ and extended perinatal mortality of 22.6 per 1000 births (14 out of 620).

The researchers said that all perinatal deaths following SARS-CoV-2 infection in pregnancy occurred in those who were unvaccinated at the time of infection, but they did not have access to detailed clinical records to assess whether covid-19 "directly or indirectly contributed to the preterm births and deaths.”

In comparison, women who received the covid-19 vaccine during pregnancy (both those who did and did not become infected with SARS-CoV-2) had a preterm birth rate of $9 \%$ (495 of 5752 live births) and an extended perinatal mortality of 4.3 per 1000 births (25 of 5766 births). The researchers said these were similar levels to the background preterm birth rate (8\%) and extended perinatal mortality (5.6 per 1000 births).

Royal College of Obstetricians and Gynaecologists president Edward Morris said, "While this study looks at the outcomes of births in Scotland, it reflects what we are seeing nationally - that contracting covid-19 while pregnant can lead to an increased risk of having a stillbirth and the baby being born prematurely. We want to reassure women that the vaccine and booster are safe in pregnancy and it is the best way of protecting you and your baby from covid-19."

Stock SJ, Carruthers J, Calvert C, et al. SARS-CoV-2 infection and covid-19 vaccination rates in pregnant women in Scotland. Nat Med 2022. doi: 10.1038/s41591-021-01666-2. pmid: 35027756

2 Mahase E. Covid-19: Pregnant women with virus are more likely to need intensive care, study finds. BMJ2020;370:m3391. doi: 10.1136/bmj.m3391 pmid: 32878867

3 lacobucci G. Covid-19 and pregnancy: vaccine hesitancy and how to overcome it. BM/2021;375:n2862. doi: 10.1136/bmj.n2862 pmid: 34810161

This article is made freely available for personal use in accordance with BMJ's website terms and conditions for the duration of the covid-19 pandemic or until otherwise determined by BMJ. You may download and print the article for any lawful, non-commercial purpose (including text and data mining) provided that all copyright notices and trade marks are retained. 